\title{
Effects of Fatigue on Throwing Performance in Experienced Team Handball Players
}

\author{
by \\ Alberto Nuño ${ }^{1}$ Ignacio J. Chirosa ${ }^{1}$, Roland van den Tillaar2, Rafael Guisado4, \\ Ignacio Martín ${ }^{3}$, Isidoro Martinez ${ }^{5}$, Luis J. Chirosa ${ }^{1}$
}

The purpose of this study was to investigate the effect of central and peripheral physiological fatigue on throwing accuracy and ball release velocity in team handball. Twenty male subjects (age $24.7 \pm 3.9$ yrs, body mass 88.5 $\pm 5.0 \mathrm{~kg}$, body height $1.86 \pm 0.05 \mathrm{~m}$, training experience $12.7 \pm 3.8 \mathrm{yrs}$ ) from one handball team participated in this study. The participants completed four sets of eight laps of a circuit that consisted of specific team handball drills/exercises, with decreasing recovery times between the laps in each set in order to induce physiological fatigue. Duration of the recovery intervals determined the description of the effort made in each set: "light" (80 s recovery between laps), "moderate" (40 s), "hard" (20 s) and "very hard" (10 s). A heart rate, concentration of lactate in blood and the rate of perceived exertion (RPE) were recorded. Ball velocity and accuracy were measured after each set and they both decreased during a fatigue protocol. However, accuracy only decreased significantly in the end of the protocol, while ball release was already affected after the first round of the protocol. The results substantiate the initial hypothesis and confirm that both throwing accuracy and ball release velocity decrease significantly as physiological fatigue increases. These variables began to decrease when the fatigue quantification values were high or very high. The findings can be used by coaches to develop training programs to teach players how to identify fatigue thresholds and combat the effects of fatigue through decision-making skills at critical game moments.

Key words: specific training, physiological fatigue, heart rate, blood lactate, rating of perceived exertion.

\section{Introduction}

Team handball is an Olympic team sport, in which physical contact is permitted and furthermore, includes throwing, sprinting, jumping, pushing and blocking actions (Gorostiaga et al., 2006; Krüger et al., 2014). It is a physically demanding sport, which is of both aerobic and anaerobic character (Michalsik et al., 2014; Rannou et al., 2001). Therefore, team handball players require well-developed physical capabilities to respond to the intense, intermittent nature of the sport. In the final phase of a match or a training session it can be observed that skills of players often decline, which is usually attributed to fatigue (Rannou et al., 2001).

From a physiological perspective, there are two types of fatigue: central fatigue, defined as the loss of performance caused by processes proximal to neuromuscular synapses, and peripheral fatigue, which is the loss of contractile force caused by distal processes (Ament and Verkeke, 2009). Both types of fatigue can appear during a match or a training session, and may produce a

\footnotetext{
1 - CTS 642 (IDAFISAD) Research Group. Department of Physical Education and Sport. University of Granada (Spain).

2 - Department of Sport Science and Physical Education of Nord University, Levanger (Norway).

3 - CTS 642 (IDAFISAD) Research Group. Department of Methodology of Behavioural Sciences. University of Granada (Spain).

4 - Department of Nursing Science. University School of Healthcare Science. University of Granada (Spain).

5 - CTS 642 (IDAFISAD) Research Group. Department of Physical Education and Sport. University of León (Spain).
} 
loss of coordination and an increased number of errors (Ament and Verkeke, 2009). Obviously this has a negative impact on game situations, especially in critical moments in the final part of a match (Michalsik et al., 2013). The effects of central and peripheral physiological fatigue may affect two very important variables in throwing performance (the main activity, besides running) in team handball i.e., throwing accuracy (van den Tillaar and Ettema, 2003a, 2006) and throwing velocity (Granados et al., 2007, 2008).

Several studies analyzing the relationship between fatigue and specific athletic skills have been conducted applying validated, but nonspecific tests and using instruments such as a cycle ergometer or a treadmill to induce fatigue (Davey et al., 2002; McMorris, et al., 2005). It was then concluded that neither throwing accuracy nor decision making were impaired by fatigue, however, ball velocity decreased as fatigue increased (Royal et al., 2006; Stevens et al., 2010). Although these exercises induced fatigue in athletes, their non-specific nature may have prevented them from experiencing a mental state comparable to that achieved in a real-competition situation.

On the other hand, more sport-specific fatigue protocols have been also used to induce fatigue to investigate its effect on different motor abilities in athletes (Michalsik et al., 2013; Zech et al., 2012). In tennis it was found that after specific incremental and intermittent effort tests, the accuracy of tennis strokes and the serve velocity decreased (Davey et al., 2002; Rota et al., 2014). In soccer the performance of specific skills (i.e. shortpassing ability) was negatively affected by fatigue (Lyons et al., 2006; Rampinini et al., 2008). However, Ferraz et al. (2012) found that firstly kicking velocity decreased due to fatigue, although it increased again after completing the last fatigue protocol. In a second study (Ferraz et al., 2016), they found that ball velocity only decreased after one round of the testing protocol and then stabilized, while accuracy did not change. They concluded that these results were probably caused by the mental/motivational concept of "pacing" and, moreover, that performance could be variable and might be dependent on self-regulation of the effort of the players according to some conscious and subconscious factors (Ferraz et al., 2012). In another study in which soccer players had to complete a circuit with different intensities, Ferraz et al. (2015) found that only at the highest intensity kicking velocity decreased, while at lower intensities no effect on ball velocity and accuracy was found.

To our knowledge, in team handball, no studies on the relationship between fatigue and specific motor skills, especially throwing performance, have been published. Therefore, the purpose of this study was to investigate the effects of fatigue on throwing ball release velocity and throwing accuracy in team handball players.

\section{Material and Methods}

\section{Participants}

Twenty male subjects (age $24.7 \pm 3.9$ yrs, body mass $88.5 \pm 5.0 \mathrm{~kg}$, body height $1.86 \pm 0.05 \mathrm{~m}$, training experience $12.7 \pm 3.8 \mathrm{yrs}$ ) from one handball team participated in this study. Of the 20 participants, 12 were professional players in the club's highest ranked team in the Second Division of the Spanish League. The other eight participants belonged to the junior team of the same club and were amateur players. All 20 participants had over eight year experience playing team handball. The subjects were fully informed about the protocol before participating in this study. Informed consent was obtained prior to all testing from the subjects, the study was conducted in accordance with the recommendations of the ethics committee of the University of Granada and current ethical standards in sports and exercise research.

\section{Procedures}

At the start of the test, prior to a warm up, the participants remained seated quietly for two minutes. Then a resting heart rate was measured, using a FC Polar S620 monitor (Polar Kempeler, Oy, Finland) and a resting blood lactate level was determined, using a Lactate Pro analyzer (KDK Array, Japan), from a $5 \mathrm{ml}$ sample of capillary blood from the right ear lobe. After these baseline measurements, a 15 min warm-up was performed. It included general and specific team handball movements, such as calisthenics, running, short sprints, passes to teammates at specific distances and throws at the goal. At the end of the warm-up, the participants performed three sets of six throws toward the openings in the customized goal. 
After the warm-up, each subject performed three throws at the goal from a distance of $7 \mathrm{~m}$ in order to record their maximal ball release velocity. The fastest throw was taken as the maximum throw velocity reference value. Subsequently, the first measurement of blood lactate concentration was taken straight after the three throws.

Afterwards, the participants began the first circuit of the testing protocol. A circuit composed of specific team handball skills was designed to induce fatigue since numerous studies had shown that data obtained from sport-specific tests were more closely related to competition than nonspecific tests (Coutts et al., 2009; Hill-Haas et al., 2010; Kelly and Drust, 2009). The participants completed four sets of eight laps of the circuit, with decreasing recovery times between the laps in each set (Royal et al., 2006) in order to induce both central and peripheral physiological fatigue. Duration of the recovery intervals determined the description of the effort made in each set: "light" (80 s recovery between laps), "moderate" (40 s), "hard" (20 s) and "very hard" (10 s). These recovery intervals were determined on the basis of the results of a preliminary study carried out with three professional team handball players, using the Borg rating of perceived exertion scale (Borg et al., 1987). The participants were instructed and verbally encouraged during the performance of the circuit to make maximum possible effort during each lap. In order to ensure the participants exerted maximum effort in the repetitions, the duration of each lap was recorded, together with the participants' heart rate. Between each set the participants were allowed $3 \mathrm{~min}$ of rest for recovery (Figure 1).

The team handball circuit was carried out in an area measuring $12 \times 12 \mathrm{~m}$, in the center of a team handball court of official dimensions (Figure 2). The circuit consisted of the following exercises: 10 push-ups with palms open and separated to elbow width, a $12 \mathrm{~m}$ run while dribbling a ball, a $15 \mathrm{~m}$ run with long and short passes to a teammate who remained standing, defensive $6 \mathrm{~m}$ runs with forward and backward movements of 2 $\mathrm{m}$; a $6 \mathrm{~m}$ run with four changes of direction touching training cones and a diagonal $15 \mathrm{~m}$ sprint.

Immediately after each set, the participants were instructed to throw with a regular handball (circumference of $58 \mathrm{~cm}$ and weight of $0.425 \mathrm{~kg}$ ) nine times with maximal effort from $7 \mathrm{~m}$ distance toward the customized goal (Figure 2). The first six times the participants were asked to throw the ball in each of the $30 \mathrm{~cm}$ circular openings in a customized shooting target covering the goal, beginning throws with the upper right hand corner (Royal et al., 2006) and proceeding clockwise. Red tape was placed around each of the six openings, forming a square measuring $40 \mathrm{x}$ $40 \mathrm{~cm}$ (Figure 2) to highlight the openings. After these six throws, three throws without taking accuracy into account to establish maximal ball release velocity were measured with a radar gun (Stalker Radar, Plano, TX, USA) with $\pm 0.028 \mathrm{~m} \cdot \mathrm{s}^{-1}$ accuracy within a field of 10 degrees from the gun. The radar gun was located $1 \mathrm{~m}$ behind the goal at the height of the thrower's shoulder.

To ensure consistency among the participants, throwing accuracy was measured by assigning points for each throw according to the way the ball entered the target. Three values were used since in a match the ball will either enter cleanly, via a crossbar, the goalkeeper or the floor, or will be saved by the goalkeeper. Therefore, the following scale was used: when the ball entered cleanly the target: 10 points; when the ball hit the target and bounced from the edges of the opening or the ball did not enter, but touched the area covered by the red strip: 5 points; when the ball neither entered nor touched the area covered by the red strip: 0 points.

After completing each set, the heart rate and ratings of perceived exertion (RPE) were measured. Furthermore, the heart rate was measured just before the participant started each circuit. These variables were chosen to measure fatigue since they had been widely used to accurately measure both central and peripheral components of fatigue (Borg et al., 1987; Mercer, 2001). The RPE was recorded on a Borg scale from 0 to 10 points (Borg et al., 1987; Foster et al., 2010; Hill-Hass et al., 2010). Due to the invasive nature of the test, blood lactate was only recorded at the end of the second set and at the end of the test.

All tests were performed at the beginning of the season between weeks five and six (week 34 of September), before the competition period started. Goalkeepers were excluded from our study. One week before the experimental phase, each athlete participated in a throwing accuracy training session which consisted of five sets of six 
throws. Throwing order was the same as on the test day, for both left and right handed players (Royal et al., 2006).

\section{Statistical Analyses}

All the variables were expressed as a mean \pm standard deviation. Normality distribution of the variables was assessed using a KolmogorovSmirnov test. To compare the effects of fatigue, a one -way ANOVA with repeated measures (5 levels: pre and after each circuit) for each variable was used. When the assumption of Mauchly sphericity was violated, a Greenhouse-Heisser method was used. The Bonferroni post hoc method was applied to analyze the significant differences. Effect size was evaluated with $\eta^{2} \mathrm{p}$ (partial Eta squared) where, according to Cohen (1988), $0.01<\eta 2<0.06$ constituted a small effect, $0.06<\eta 2<0.14$ a medium effect and $\eta 2>0.14$ a large effect. For all statistical calculations, SPSS version 16.0 (SPSS Inc, Chicago, IL) was used. The level of significance was set at $p<0.01$.

\section{Results}

Maximal ball release velocity $(F=24.7, p<$ $\left..001, \eta^{2}=0.57\right)$ and throwing accuracy $(F=123.5, p$ $\left.<0.001, \eta^{2}=0.867\right)$ were affected significantly after completing the circuits with different recovery pauses. Post hoc comparisons showed that ball release velocity decreased significantly after the first circuit $(-4.5 \%)$ compared with pretest values. Then, it increased significantly again, although it decreased from the second to the third (-4.9\%) and further from the third $(-7 \%)$ to the last circuit (Figure 4). Throwing accuracy decreased significantly after the third circuit (-37\%) and again after the fourth $(-73 \%)$ and the last circuit (Figure 4).

The heart rate increased every lap in each circuit $\left(F=267, p<.001, \eta^{2} \geq .93\right.$, Figure 5) and when measured before the start $(F=720, p<.001$, $\left.\eta^{2}=.97\right)$ and after each circuit $(F=1423 ; p<0.001$; $\left.\eta^{2}=0.987\right)$, it also increased significantly over the exercise period (Figure 6). This was also accompanied by significant increases after each circuit in the rate of perceived exertion ( $\mathrm{F}=403$; $p$ $<0.001 ; \eta^{2}=0.955$; Figure 5). Furthermore, lactate concentration also increased significantly in each measurement during the protocol $(F=1183, p<$ $.001, \eta^{2}=.98$, Figure 5). Post hoc comparison showed that the heart rate, the RPE and lactate concentration increased significantly at every measurement after each circuit.

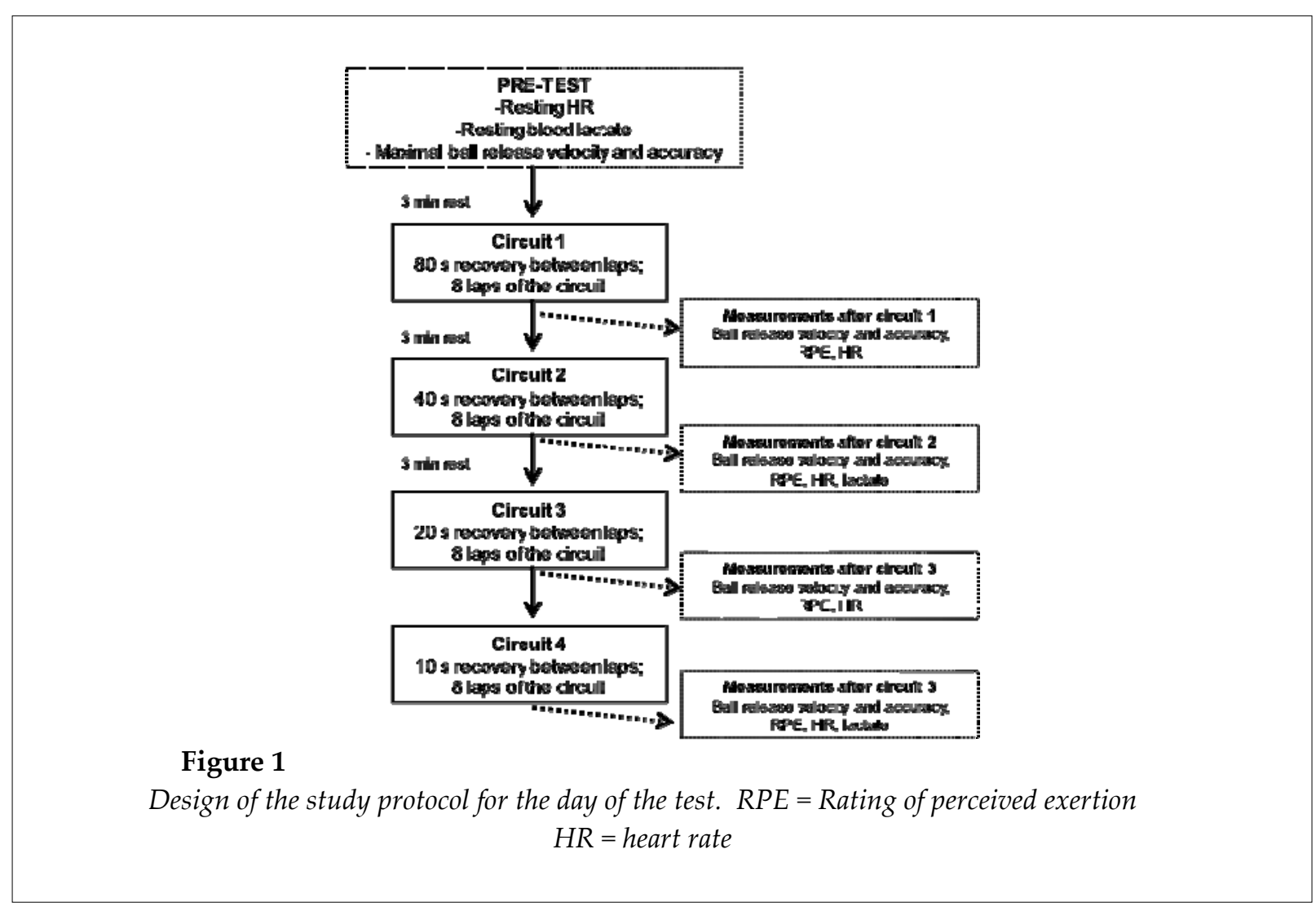




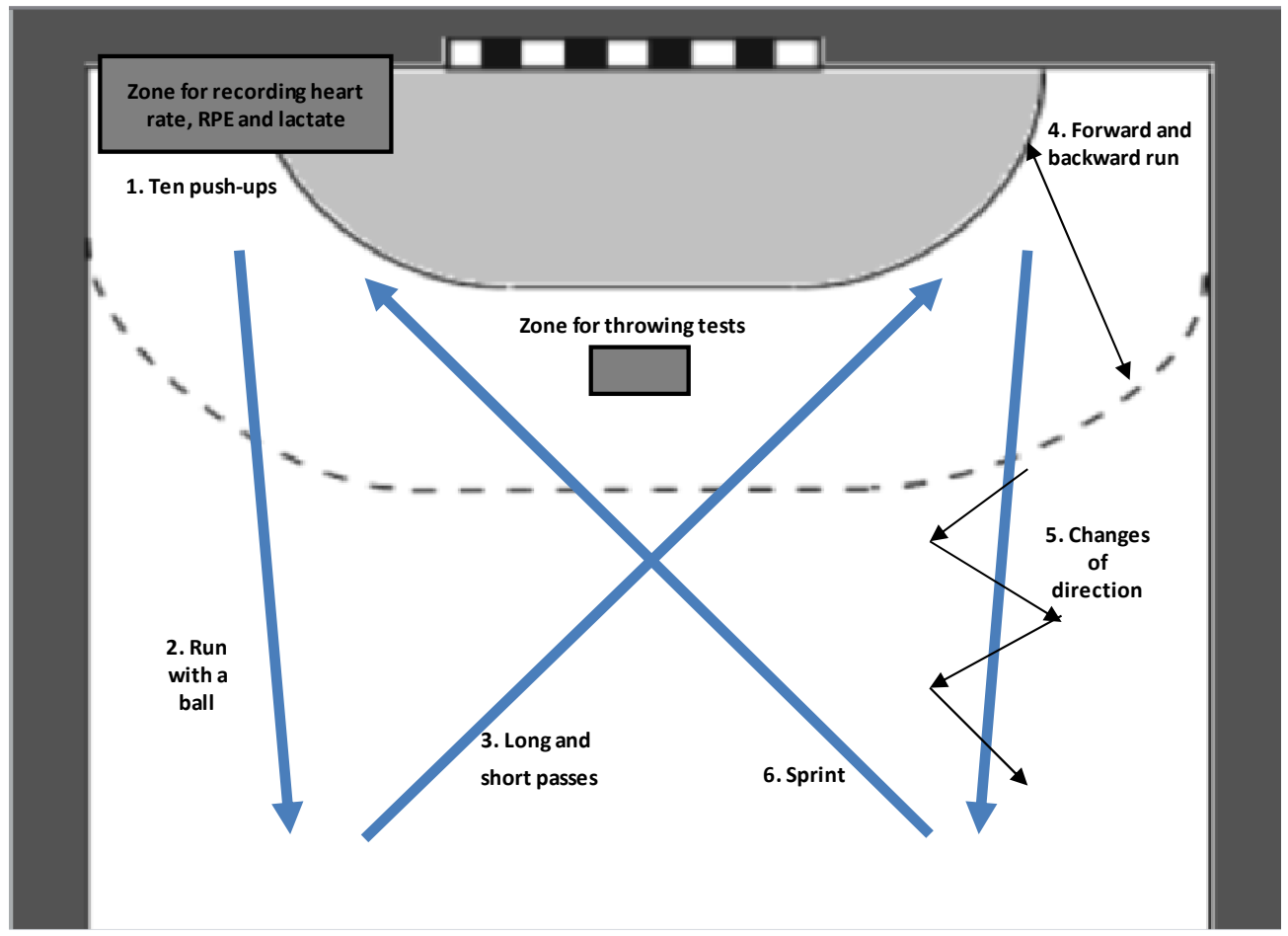

Figure 2

Diagram of the team handball circuit, data recording areas and the area for the throwing accuracy and ball release velocity tests.

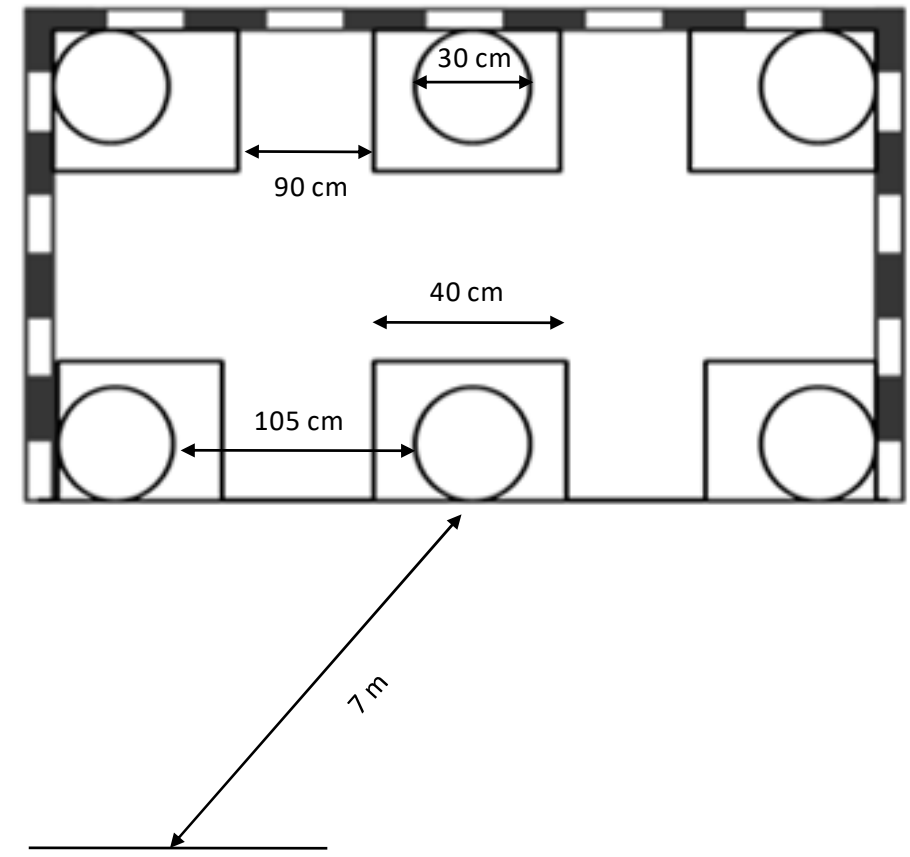

Figure 3

Goal with six openings (targets) to measure throwing accuracy in team handball. 

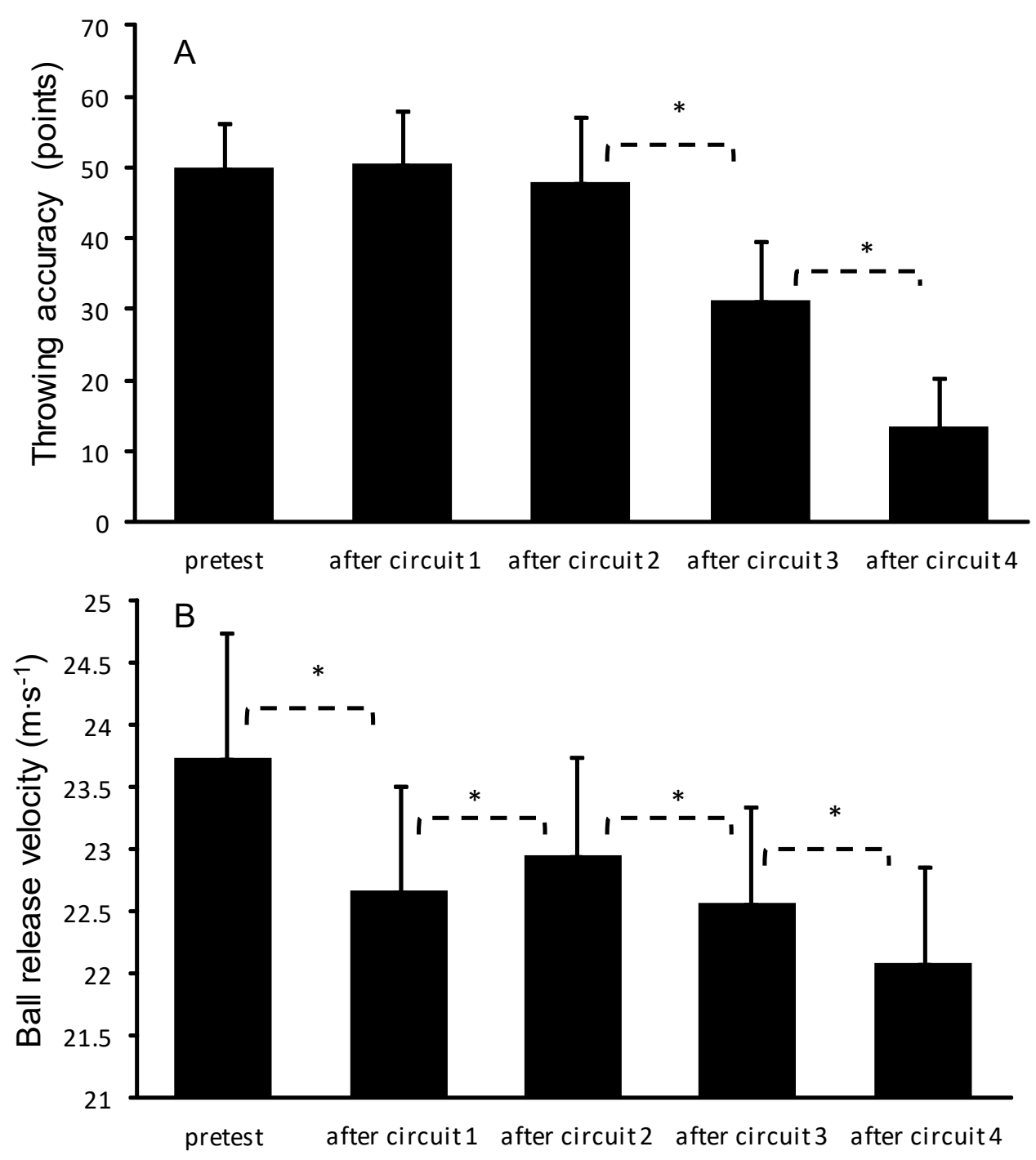

Figure 4

Mean (SD) throwing accuracy $(A)$ and ball release velocity $(B)$ at the pretest and after each circuit.

* indicates a significant difference on a .01 level between these two conditions. 

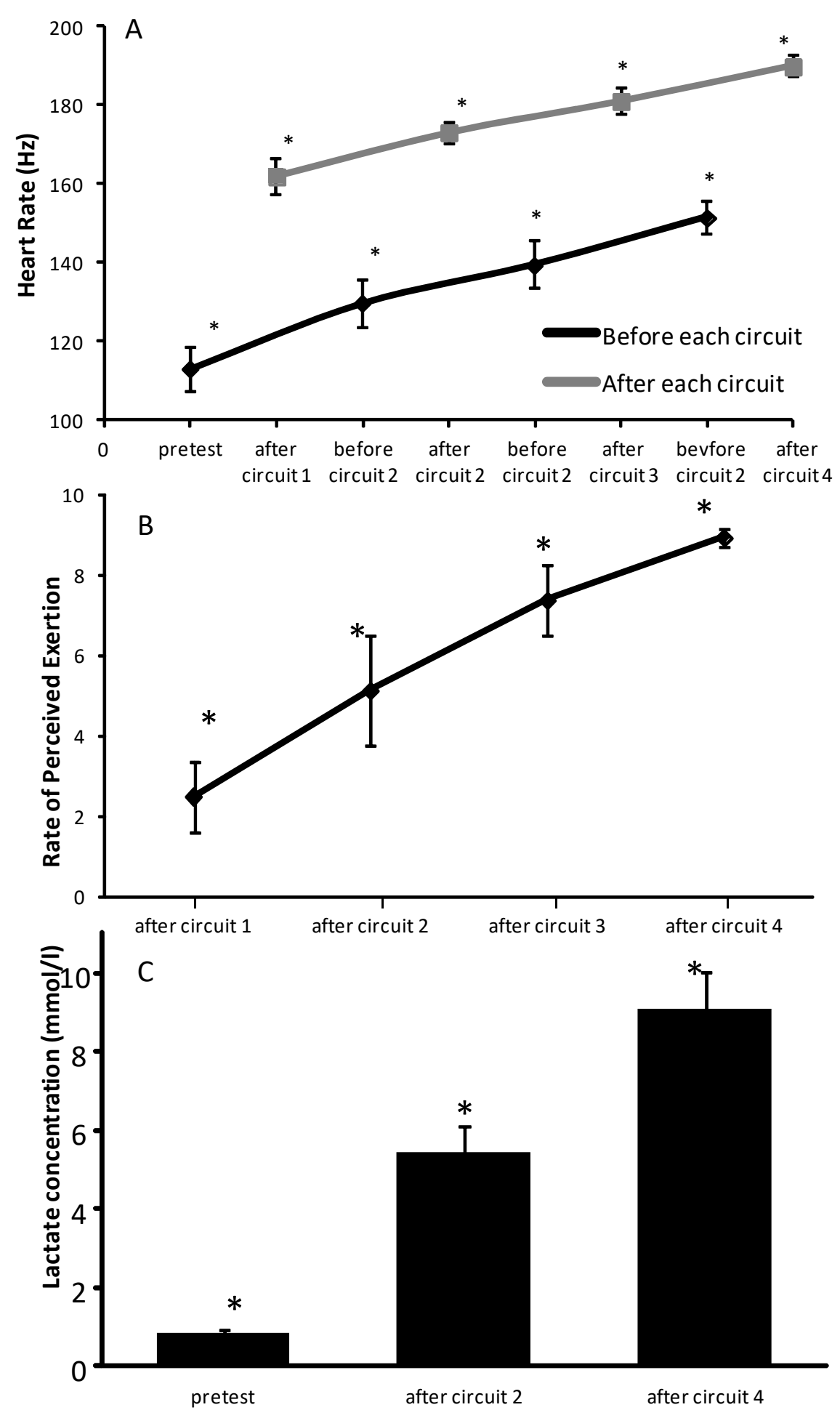

Figure 5

Mean (SD) heart rate (A), rating of perceived exertion (RPE) (B) and lactate concentration at different point times during testing.

* indicates a significant difference on a .01 level of this variable compared to all others. 


\section{Discussion}

The main objective of this study was to analyze the effect of fatigue on throwing performance in team handball players. Throwing velocity and accuracy were measured and both components were affected as recovery time was reduced between circuits (Figure 4).

The ball release velocity declined already after the first circuit. This was in line with the findings of Ferraz et al. (2012, 2016) in soccer kicking. They also used a specific circuit five times to provoke fatigue. After the second circuit, an increase in ball release velocity was found in our study, which was also in line with the soccer kicking study by Ferraz et al. (2012). In their study, an increase in ball velocity after the third circuit was observed. They explained this phenomenon by the central governor model and the concept of "pacing" (Noakes et al., 2004). It can be stated that the intensity of the first circuit causes an increase in metabolic stress, thereby increasing the heart rate and the RPE in the participant and ball release velocity declines due to this change. In the second circuit, the subject knows how tiring the circuit is and starts "pacing". Thereby, throwing velocity increases again. However, Ferraz et al. (2012) showed that ball release velocity increased further after the third circuit, while the opposite occurred in our study. An explanation for this is that we used a progressive fatigue protocol shortening the recovery periods (80-40-20-10 s rest between each lap), while Ferraz et al. (2012) just continued with the same circuit without any change of duration in the rest periods. This difference in protocols between the studies causes that the subjects in the study of Ferraz et al. (2012) could use pacing and the reserve for muscle recruitment (the security reserve) that can be used for the so called "end spurt" at the highest level of peripheral fatigue (Millet, 2011). Edwards and Noakes (2009) showed that players were well known to selfregulate match-play efforts following numerous extrinsic and intrinsic factors. They suggested that athletes modulated effort according to a subconscious strategy. As such, subconscious factors influence conscious behavioral decisions to regulate effort. Consequently, as shown in our study, we suggest that players may have self regulated their effort (pacing strategy) throughout the start of the protocol.
However, in our study the rest period between the laps was shortened for each circuit (80-40-20-10 s), which made it difficult for the subjects to continue with pacing. This was clearly shown by the heart rates, lactate concentration and the RPE (Figure 5) in our study. These variables increased significantly after each circuit, while in the study of Ferraz et al. (2012) the observed increase was smaller. Furthermore, due to these shorter resting periods, the reserve for muscle recruitment will be emptied quicker (Millet, 2011). This is probably the cause for the decrease of maximal ball release velocity after circuits three and four (Figure 4).

The development of throwing accuracy did not follow the same development as the ball release velocity; the accuracy started to decrease after the third circuit and continued after conducting the last circuit (Figure 4). These results differed from the ones obtained in similar studies performed on water polo players (Royal et al., 2006; Stevens et al., 2010) and soccer players (Ferraz et al., 2015), in which it was concluded that throwing accuracy did not vary with fatigue. In the water polo studies, accuracy improved notably by the end of the test. However, it should be also noted that technical components of those throws may have been neglected in order to keep accuracy constant. This was attributed to the selfregulation skill level of the athletes allowing them to have optimal performance in fatigued conditions. In our case, the participants presented varied skill levels (resulting from competing in different leagues), which may have impacted the overall statistics regarding self-regulation and compensation for fatigue (Royal et al., 2006). Despite this, other studies carried out with soccer players (Coutts et al., 2009; Hill-Haas et al., 2010; Kelly and Drust, 2009) and tennis players (Davey et al., 2002) have confirmed that accuracy is indeed affected when fatigue reaches high values. Thus, both ball throwing performance variables are affected by fatigue generated in a handball specific circuit.

It is necessary to highlight the importance that this specifically designed circuit has for team handball training, considering its aim to produce and maintain a state of physiological fatigue among the participants of this study. Previous research demonstrated that when well-trained subjects participated in sport-specific tests or 
circuits, their neuromuscular systems behaved following patterns similar to competition, but this was not the case when non-specific tests were used (Gabbet, 2010; Magalhaes et al., 2010). In many other studies, the type of intervention used to induce fatigue is based on non-specific running and/or pedalling protocols. Although these are effective at provoking fatigue, they create a sensory state that is different from that experienced during competition. In the present study, however, a specific task was designed to simulate team handball playing situations and recording methods were standardized. Thus, the effort was accurately measured and the specificity of the stimulus was assured (Castagna et al., 2010; Gabbet, 2010). Fatigue was produced by reducing the recovery time allowed between sets (Royal et al., 2006).

There are some limitations in the present study which should be noted. Firstly, the subjects were note elite players (the second division of the Spanish Handball League) which could influence the results due to their training background. Future studies should include subjects of the First Division (ASOBAL) or from other clubs involved in the EHF leagues to determine if the results are similar for elite players. Secondly, as noted by Ferraz et al. $(2012,2016)$ in soccer kicking, it seems that players are able to self-regulate during games by employing the "pacing strategy" (Noakes, 2004). Furthermore, as indicated by Ferraz et al. (2012, 2016), players' awareness of the total number of sets in a series may influence the results. Additionally, from our point of view, a repeated measures design is not the most appropriate for data collection. By the last lap of a circuit, players had accumulated a lot of fatigue and did not really know whether it was produced as a result of decreased recovery time or the accumulation of laps of the specific circuit. Therefore, future research should address this issue from the perspective of the experimental design between groups.

In the present study, we only investigated the effect of fatigue on throwing performance (accuracy and velocity). It is probable that changes in coordination occurred (van den Tillaar and Ettema, 2003b) and that they caused a decrease in throwing performance. These changes in coordination could also influence other technical-tactical responses of participants under competition-like situations. Therefore, further research should be conducted to investigate which changes in coordination in particular cause the decreases in throwing performance. Furthermore, it would be interesting to find out if these coordination changes also influence other technical-tactical responses of participants.

The main conclusion of our study is that the greater the fatigue (induced by reduced recovery times and accumulation of series of specific circuits), the worse the performance (ball release velocity and accuracy) in ball throwing in handball players. Coaches and trainers should take these important findings into account when modulating training intensity, especially when the goal of the session is of a technical and tactical nature, considering that the results of our study indicate that the shorter the recovery between high intensity sets of physical work performed, the greater the loss of throwing velocity and accuracy. Therefore, in training after bouts of high intensity work, recovery of at least 40 seconds is suggested to preserve the quality of throwing accuracy and velocity.

\section{Acknowledgements}

The authors thank the Málaga Aldemar Team handball Club and the Málaga Maristas Club for their collaboration and support. We also express our appreciation for the confidence shown in us and the commitment made by the trainers of the various teams and by the players who took part in this study. No financial support was received for this study.

\section{References}

Ament W, Verkerke GJ. Exercise and fatigue. Sports Med, 2009; 39: 389-422

Borg G, Hassmén P, Lagerström M. Perceived exertion related to heart rate and blood lactate during arm and leg exercise. Eur J Appl Physiol Occup Physiol, 1987; 56: 679-685

Castagna C, Manzi V, Impellizzeri F, Weston M, Barbero-Alvarez JC. Relationship between endurance field 
tests and match performance in young soccer players. J Strength Cond Res, 2010; 24: 3227-3233

Chelly MS, Hermassi S, Shephard RJ. Relationships between power and strength of the upper and lower limb muscles and throwing velocity in male handball players. J Strength Cond Res, 2010; 24: 1480-1487

Coutts AJ, Rampinini E, Marcora SM, Castagna C, Impellizzeri FM. Heart rate and blood lactate correlates of perceived exertion during small-sided soccer games. J Sci Med Sport, 2009; 12: 79-84

Davey PR, Thorpe RD, Williams C. Fatigue decreases skilled tennis performance. J Sports Sci, 2002; 20: 311318

Ferraz R, van den Tillaar R, Marques MC. The effect of fatigue upon kicking velocity in soccer players. J Hum Kinet, 2012; 35: 89-99

Ferraz R, van den Tillaar R, Marques M. The effect of different intensities upon kicking accuracy and velocity in soccer players. J Sports Health Sci, 2015; Ahead online

Ferraz R, Pereira A, van den Tillaar R, Marques M. The effect of fatigue and the knowledge of duration upon kicking accuracy and velocity in soccer players. J Sports Health Sci, 2016; Ahead online

Foster CD, Twist C, Lamb KL, Nicholas CW. Heart rate responses to small-sided games among elite junior rugby league players. J Strength Cond Res, 2010; 24: 906-911

Gabbett TJ. The development of a test of repeated-sprint ability for elite women's soccer players. J Strength Cond Res, 2010; 24: 1191-1194

Gorostiaga EM, Granados C, Ibañez J, Izquierdo, M. Differences in physical fitness and throwing velocity among elite and amateur male handball players. Int J Sports Med, 2005; 26: 225-232

Gorostiaga EM, Granados C, Ibañez J, Gonzalez-Badillo JJ. Izquierdo M. Effects of an entire season on physical fitness changes in elite male handball players. Med Sci Sports Exerc, 2006; 38: 357-366

Granados C, Izquierdo M, Ibañez J, Bonnabau H, Gorostiaga EM. Differences in physical fitness and throwing velocity among elite and amateur female handball players. Int J Sports Med, 2007; 28: 860-867

Granados C, Izquierdo M, Ibañez J, Ruesta M, Gorostiaga EM. Effects of an entire season on physical fitness in elite female players. Med Sci Sports Exerc, 2008; 40: 351-361

Hermassi S, Chelly MS, Fathloun M, Shephard RJ. The effect of heavy- vs. moderate-load training on the development of strength, power, and throwing ball velocity in male handball players. J Strength Cond Res, 2010; 24: 2408-2481

Hill-Haas SV, Coutts AJ, Dawson BT, Rowsell GJ. Time-motion characteristics and physiological responses of small-sided games in elite youth players: the influence of player number and rule changes. $J$ Strength Cond Res, 2010; 24: 2149-2156

Impellizzeri FM, Rampinini E, Coutts AJ, Sassi A, Marcora SM. Use of RPE-based training load in soccer. Med Sci Sports Exerc, 2004; 36: 1042-1047

Kelly DM, Drust B. The effect of pitch dimensions on heart rate responses and technical demands of smallsided soccer games in elite players. J Sci Med Sport, 2009; 12: 475-479

Kristensen GO, van den Tillaar R, Ettema G. Velocity specificity in early-phase sprint training. J Strength Cond Res, 2006; 20: 833-837

Krüger K, Pilat C, Uckert K, Frech T, Mooren FC. Physical performance profile of handball players is related to playing position and playing class. J Strength Cond Res, 2014; 28: 117-125

Lyons M, Al-Nakeeb Y, Nevill A. Performance of soccer passing skills under moderate and high-intensity localized muscle fatigue. J Strength Cond Res, 2006; 20: 197-202

Magalhaes J, Rebelo A, Oliveira E, Silva J, Marques F, Ascensao A. Impact of Loughborough Intermittent Shuttle Test versus soccer match on physiological, biochemical and neuromuscular parameters. Eur J Appl Physiol, 2010; 108: 39-48

Marques MC, van den Tillaar R, Vescovi JD, Gonzalez-Badillo JJ. Relationship between throwing velocity, 
muscle power, and bar velocity during bench press in elite handball players. Int J Sports Physiol Perform, 2007; 2: 414-422

McMorris T, Delves S, Sproule J, Lauder M, Hale B. Effect of incremental exercise on initiation and movement times in a choice response, whole body psychomotor task. Br J Sports Med, 2005; 39: 537541

Mercer TH. Reproducibility of blood lactate-anchored ratings of perceived exertion. Eur J Appl Physiol, 2001; 85: 496-499

Michalsik LB, Madsen K, Aagaard P. Physiological capacity and physical testing in male elite team handball. J Sports Med Phy Fitness, 2015; 55: 415-429

Michalsik LB, Aagaard P, Madsen K. Locomotion characteristics and match-induced impairments in physical performance in male elite team handball players. Int J Sports Med, 2013; 34: 590-599

Rampinini E, Impellizzeri FM, Castagna C, Azzalin A, Bravo DF, Wisloff U. Effect of match-related fatigue on short-passing ability in young soccer players. Med Sci Sports Exerc, 2008; 40: 934-942

Rannou F, Prioux J, Zouhal H, Gratas-Delamarche A, Delamarche P. Physiological profile of handball players. J Sports Med Phys Fitness, 2001; 41: 349-353

Rota S, Morel B, Saboul D, Rogowski I, Hautier C. Influence of fatigue on upper limb muscle activity and performance in tennis. Journal of Electromyography and Kinesiology, 2014; 24: 90-97

Royal KA, Farrow D, Mujika I, Halson SL, Pyne D, Abernethy B. The effects of fatigue on decision making and shooting skill performance in water polo players. J Sports Sci, 2006; 24: 807-815

Stevens HB, Brown LE, Coburn JW, Spiering BA. Effect of swim sprints on throwing accuracy and velocity in female collegiate water polo players. J Strength Cond Res, 2010; 24: 1195-1198

van den Tillaar R, Ettema G. Influence of instruction on velocity and accuracy of overarm throwing. Percept Mot Skills, 2003a; 96: 423-434

van den Tillaar R, Ettema G. Instructions emphasizing velocity, accuracy, or both in performance and kinematics of overarm throwing by experienced handball players. Percept Mot Skills, 2003; 97: 731-742

van den Tillaar R, Ettema G. A comparison between novices and experts of the velocity-accuracy trade-off in overarm throwing. Percept Mot Skills, 2006; 103: 503-514

van den Tillaar R, Ettema G. A comparison of overarm throwing with the dominant and nondominant arm in experienced team handball players. Percept Mot Skills, 2009a; 109: 315-326

van den Tillaar R, Ettema G. Is there a proximal-to-distal sequence in overarm throwing in team handball? J Sports Sci, 2009b; 27: 949-955

Zech A, Steib S, Hentschke C, Eckhardt H, Pfeifer K. Effects of localized and general fatigue on static and dynamic postural control in male team handball athletes. J Strength Cond Res, 2012; 26: 1162-1168

\section{Corresponding author:}

\section{Ignacio J. Chirosa}

Department of Physical Education and Sport,

University of Granada (Spain)

Carretera de Alfacar S/N 18011

Phone: +34 958246979

Fax: +34958244369

E-mail: ichirosa@ugr.es 